\title{
Short communication: Greater intensity of estrous expression is associated with improved embryo viability from superovulated Holstein heifers
}

\author{
A. M. L. Madureira, ${ }^{1} \odot$ T. A. Burnett, ${ }^{1} \odot$ K. G. Pohler, ${ }^{2}$ T. G. Guida, ${ }^{3}$ C. P. Sanches Jr. $^{3}$ J. L. M. Vasconcelos, ${ }^{3} \odot$ \\ and R. L. A. Cerri ${ }^{1 *}$ (1) \\ ${ }^{1}$ Applied Animal Biology, Faculty of Land and Food Systems, University of British Columbia, Vancouver, Canada V6T 1 Z4 \\ ${ }^{2}$ Department of Animal Science, Texas A\&M University, College Station 77843 \\ ${ }^{3}$ Department of Animal Production, São Paulo State University, Botucatu, Brazil 18168-000
}

\section{ABSTRACT}

The aim of this study was to determine the association between estrous expression, measured using a breeding indicator and an automated activity monitor (AAM), and the success of embryo collection after superovulation. Holstein heifers $(\mathrm{n}=51 ; 10.5$ to $14.5 \mathrm{mo}$, and $325.0 \pm 21.1 \mathrm{~kg}$ of body weight) were superovulated ( $\mathrm{n}=69$ events) for the collection of embryos using a protocol based on sequential administration of FSH for follicle superstimulation and $\mathrm{GnRH}$ to induce ovulation. Artificial insemination (AI) was performed twice, once at the moment of GnRH administration and again $12 \mathrm{~h}$ later, using thawed, sexed semen. Ovaries were scanned via ultrasonography on the day of the first AI to count the total number of preovulatory follicles and $7 \mathrm{~d}$ later for the total number of corpora lutea present. Embryos were collected $7 \mathrm{~d}$ post-AI, counted, and assessed for viability. A breeding indicator (Estrotect, Rockway Inc., Spring Valley, WI) and a collar-mounted AAM (CowScout Activity Monitoring System, GEA, Dusseldorf, Germany) were used to measure standing mounts and an algorithmic estimate of estrous expression, respectively. A score for the breeding indicator was given as follows: score $1=100 \%$ of the indicator was intact; score $2=50 \%$ of the indicator was rubbed off; score $3=$ greater than $50 \%$ of the indicator was rubbed off. Estrous expression detected by the AAM was quantified through the relative increase in physical activity and duration of time spent above a set threshold. Data were analyzed by ANOVA using the MIXED procedures of SAS (SAS Institute Inc., Cary, NC). The number of follicles present at AI was not affected by estrous expression. The mean $( \pm \mathrm{SD})$ ovulatory response was $67.5 \pm 26.3 \%$. We found an effect of estrous

Received October 21, 2019.

Accepted January 13, 2020.

*Corresponding author: ronaldo.cerri@ubc.ca expression as detected by the breeding indicator on the ovulatory response $(42.1 \pm 8.0$, vs. $78.2 \pm 9.0$, vs. 74.0 $\pm 4.9 \%$, for scores 1,2 , and 3 , respectively) but not from the AAM. Heifers that had a score of 3 (versus those with scores of 1 and 2) on the breeding indicator had a greater number of embryos $(4.1 \pm 0.5$, vs. $1.2 \pm$ 1.0 , vs. $1.8 \pm 1.0$ embryos), and a greater percentage of these embryos were viable $(43.1 \pm 0.05$, vs. $35.5 \pm$ 0.1 , vs. $34.3 \pm 0.1 \%$ ). Similarly, heifers that showed a greater intensity of activity (as measured by the AAM) had a greater number of embryos collected (10.2 \pm 1.2 vs. $6.0 \pm 1.3$ embryos), and a greater percentage of those embryos were viable (53.1 \pm 5.0 vs. $23.4 \pm 5.1 \%$ ). Longer-duration estrus episodes were associated with a higher percentage of viable embryos $(51.2 \pm 5.2$ vs. $25.3 \pm 5.3 \%$ ). In conclusion, stronger estrous intensity was associated with a greater number of total embryos collected and a greater percentage of viable embryos. These results suggest that monitoring the intensity of estrus could be used to predict superovulatory response as well as embryo quality in Holstein heifers.

Key words: embryo transfer, estrous expression, automated activity monitor

\section{Short Communication}

Superovulation was developed in the 1970s (Hasler, 2014) and can be used to accelerate gains in genetic progress (Jaton et al., 2016). The procedure involves providing a combination of hormones to superstimulate the ovarian follicles, leading to multiple ovulations (Peippo et al., 2011). Successful multiple ovulations are influenced by factors related to the donor (including parity, age, and breed) and to management (including the superovulation protocol, climate, and nutrition; Chebel et al., 2008). Factors that contribute to fertilization rates in superovulated animals include semen fertility, type of semen, and AI technician skills (Sartori et al., 2004; Schenk et al., 2006). Together these factors can influence fertilization rates and embryo production; 
the efficiency of superovulation methods has remained fairly stable in recent years, with an average yield of 6.2 embryos per superovulation event (IETS, 2013).

The intensity of estrous expression is related to cow fertility, both when spontaneous estruses are detected by automated activity monitors (AAM; Madureira et al., 2015) and when following a timed AI program (Pereira et al., 2016; Madureira et al., 2019). Similarly, the occurrence of estrus in recipient cows appears to increase pregnancy rates following embryo transfer in estradiol-based (Pereira et al., 2016) or GnRH-based protocols (Jinks et al., 2013). Furthermore, stronger displays of estrus are associated with decreased pregnancy losses after embryo transfer (Pereira et al., 2016). The reasons for this link between estrous expression and fertility are not well understood but may be associated with the hormonal milieu surrounding estrus, as both progesterone and estradiol play important roles in the final maturation of the oocyte, embryo development, and receptivity of the uterus, as well as being associated with differences in the occurrence and expression of estrus (Denis-Robichaud et al., 2018; Madureira et al., 2018). To date, limited literature exists about the effect of estrous expression on the superovulatory response and viability of embryos (Jinks et al., 2013; Larimore et al., 2015).

The main objective of this study was to evaluate the effect of the intensity of estrous expression on the viability of in vivo-produced embryos. We hypothesized that heifers that displayed greater estrous expression would yield a greater number of embryos, of which a greater proportion would be viable and freezable. This study was conducted at a commercial farm in Minas Gerais State, Brazil $\left(19^{\circ} 18^{\prime} 40^{\prime \prime} \mathrm{S}, 46^{\circ} 02^{\prime} 56^{\prime \prime} \mathrm{W}\right)$. The practices outlined in the Guide for the Care and Use of Agricultural Animals in Agricultural Research and Teaching (FASS, 1999) were used for all procedures as part of the local requirements.

A total of 51 nulliparous Holstein heifers $(\mathrm{n}=69$ events; 10.5 to $14.5 \mathrm{mo}$ of age; $325.0 \pm 21.1 \mathrm{~kg}$ of BW) were enrolled in the study 1 wk before the superovulation protocol. Heifers were housed in an open-pack compost-bedded barn. Animals were fed a TMR 3 times daily, which was pushed up 3 times daily. Water and TMR were available for ad libitum intake. At the time of enrollment, BCS was recorded (1 to 5 scale at increments of 0.25; Edmonson et al., 1989). Heifers were monitored continuously by a collar-mounted sensor (CowScout Activity Monitoring System, GEA, Dusseldorf, Germany) starting 1 wk before the superovulation protocol until the moment of timed AI, allowing for sufficient time for the AAM to create a baseline before the initiation of the protocol.
Heifers were submitted to a superovulation protocol based on FSH and progesterone, as described in Figure 1. Timed AI was performed twice, first at the moment of GnRH administration (d 9) and then $12 \mathrm{~h}$ later (d 10) using thawed, sexed semen delivered by a trained technician. All heifers were bred regardless of estrous expression. At the time of the progesterone insert removal (d 8), all heifers received a breeding indicator for detection of estrus (Estrotect, Rockway Inc., Spring Valley, WI) placed on the spine, halfway between the hip and the tail head; all heifers received both the AAM and the breeding indicator. At the day of first $\mathrm{AI}$, a score was given to the breeding indicator based on the proportion of the original color that remained on it (score $1=100 \%$ of the indicator was intact, and animals were classified as not being in estrus; score 2 $=50 \%$ of the indicator was rubbed off, and animals were classified as having displayed a moderately intense estrus; and score $3=$ greater than $50 \%$ of the indicator was rubbed off, and the animals were classified as having displayed a high intensity of estrous expression). The AAM system was equipped with a wireless antenna-receiver, located in the open-pack barn, that sent data to a processing unit for recording and analysis. The estrus event alarm was triggered when at least 3 consecutive data block-intervals of $2 \mathrm{~h}$ were above a set threshold recommended by the manufacturer. Two traits of estrous expression were calculated from the AAM: intensity of activity (maximum activity during an estrus episode) and duration (total time the animal spent with activity above the threshold) to describe the expression of estrus (Madureira et al., 2015). Ovulatory response was calculated via the number of follicles on the day of AI $(\geq 10 \mathrm{~mm})$ divided by the number of corpora lutea on the day of collection.

Heifers were flushed $7 \mathrm{~d}$ after the first AI by a transcervical procedure using a silicone Foley catheter (number 18; Solidor, Well Lead Medical Instruments, Guangzhou, China). The balloon of the Foley catheter was placed into the uterine horn ipsilateral to the CL. Approximately $500 \mathrm{~mL}$ of flushing solution (Dulbecco's modified PBS, Biodux, Campinas, Brazil) was used to recover structures into a sterile filter (WTA, Cravinhos, SP, Brazil). For heifers with CL on both ovaries, both horns were flushed as described previously. Oocytes or embryos collected were evaluated for fertilization and grade quality (G1 = excellent or good, G2 = fair, G3 = poor, and $\mathrm{G} 4=$ degenerated) as described by the International Embryo Technology Society (IETS, 2013). Each embryo was also classified by its stage of development, as follows: morula, initial blastocyst, blastocyst, or expanded blastocyst (Robertson and Nelson, 1998). The initial blastocyst and blastocyst groups were later 
combined due to low numbers of embryos in these stages, and no embryos were found in the expanded blastocyst category. After classification, embryos were placed into a straw $(0.25 \mathrm{~mm})$ with ethylene glycol solution (Holding, Biodux) for a fresh or frozen-thawed transfer to a recipient cow.

Distributions and normality tests were obtained using the Univariate procedure of the SAS software, version 9.4 (SAS Institute Inc., Cary, NC). Class variables used for analyses are as follows. The BCS categories were average $(<3.0)$ or moderate $(\geq 3.0)$. Duration of estrus was calculated as the number of hours above the alert threshold detected by the AAM and was categorized as shorter or longer using the median. Intensity of activity from the AAM was defined as the maximum activity value recorded during the estrus event, and was also categorized using the median, into moderate and greater estrous expression groups. Percentage and number of viable embryos, number of follicles, ovulatory response, and percentage of morula and blastocysts were used as continuous dependent variables and assessed with ANOVA using the MIXED procedure, with AI event as the experimental unit and heifer as a random effect. Age was used as a continuous independent variable. Breeding indicator score, BCS, and intensity and duration of estrous expression (as measured through the AAM) were used as categorical independent variables; breeding indicator score and intensity and duration of estrous expression were considered collinear variables and thus not included within the same models. Only variables with a $P$-value $<0.15$ were maintained in the final model. Differences with $P \leq 0.05$ were considered significant, and those between $0.05>P \leq 0.10$ were designated as a tendency.

The main objective of our study was to evaluate the association between estrous expression measured by a breeding indicator and AAM, on the viability of embryos produced in vivo. Increased estrous expression was associated with increased embryo production. Heifers with a score of 3 on the breeding indicator had a greater number and percentage of viable embryos compared with those with a score of 1 or 2 (Table $1 ; P<$ 0.05). Similarly, greater intensity of estrous expression detected by the AAM was also associated with a greater number $(P=0.01)$ and percentage of viable embryos $(P<0.01)$ compared with heifers expressing moderate intensity of activity. Moreover, heifers that had a longer duration of estrus, as measured using the AAM, tended to have more viable embryos $(P=0.10)$ and a greater percentage of viable embryos $(P<0.01)$ than did heifers that had shorter duration of estrus. Although the viability of embryos increased with increased estrous expression, the stage and quality of the embryos were unaffected. The total percentage of embryos at the morula, initial blastocyst, and blastocyst stages in this study were $78.9,18.6$, and $2.5 \%$, respectively.

Estradiol and progesterone are the main hormones acting on the hypothalamus to trigger estrous behavior (Woelders et al., 2014). Standing to be mounted has been the gold standard for estrus detection (Roelofs et al., 2010), but this behavior is well known to be reduced in lactating dairy cows (Rivera et al., 2010). In

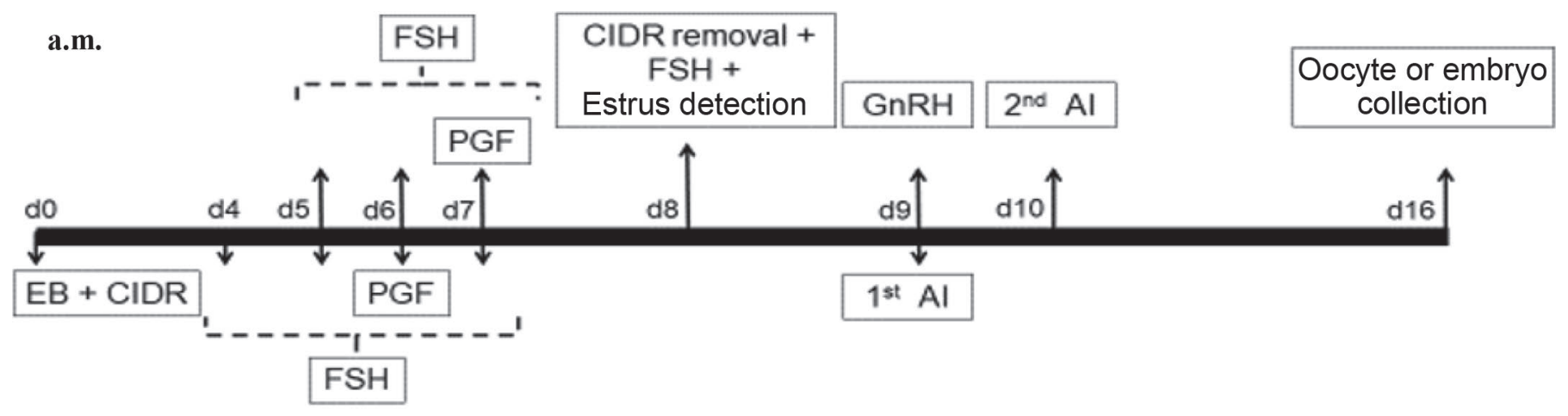

p.m.

Figure 1. The superovulation synchronization protocol used was as follows. An intravaginal progesterone implant of $1.9 \mathrm{~g}$ of progesterone previously used for $9 \mathrm{~d}$ (controlled internal drug release, CIDR; Zoetis, Sao Paulo, Brazil). A 2.0-mg (i.m.) injection of estradiol benzoate (EB; $2.0 \mathrm{~mL}$ of RIC-BE, Agener Uniao, Sao Paulo, Brazil) on p.m. of d 0. On p.m. d 4 a 2.0-mL (i.m.) injection of FSH (Folltropin, Vetoquinol, Mairiporã, Brazil). On a.m. and p.m. d 5, respectively, a 2.0- and 1.5-mL (i.m.) injection of FSH. On a.m. and p.m. d 6, respectively, a 1.5- and 1.0-mL (i.m.) injection of FSH as well as a 25-mg (i.m.) injection of dinoprost tromethamine (PGF; $5.0 \mathrm{~mL}$ of Lutalyse, Zoetis) at p.m. d 6. On a.m. and p.m. d 7, respectively, a 1.0- and 0.5-mL (i.m.) injection of FSH, and a PGF injection on a.m. d 7. On a.m. d 8 the last injection of FSH 0.5 mL (i.m.), CIDR removal, and estrus detection. On a.m. d 9 a 4-mg (i.m.) injection of Gonadorelin (GnRH; 4 mL of Fertagyl, MSD Animal Health, São Paulo, Brazil). A first AI at p.m. d 9 and second AI at a.m. d 10. On d 16, oocyte or embryo collection. 
Table 1. Number of viable embryos, percentage of viable embryos, number of follicles, and number of viable embryos per follicle according to breeding indicator score and automated activity monitor (AAM) measures of estrous expression

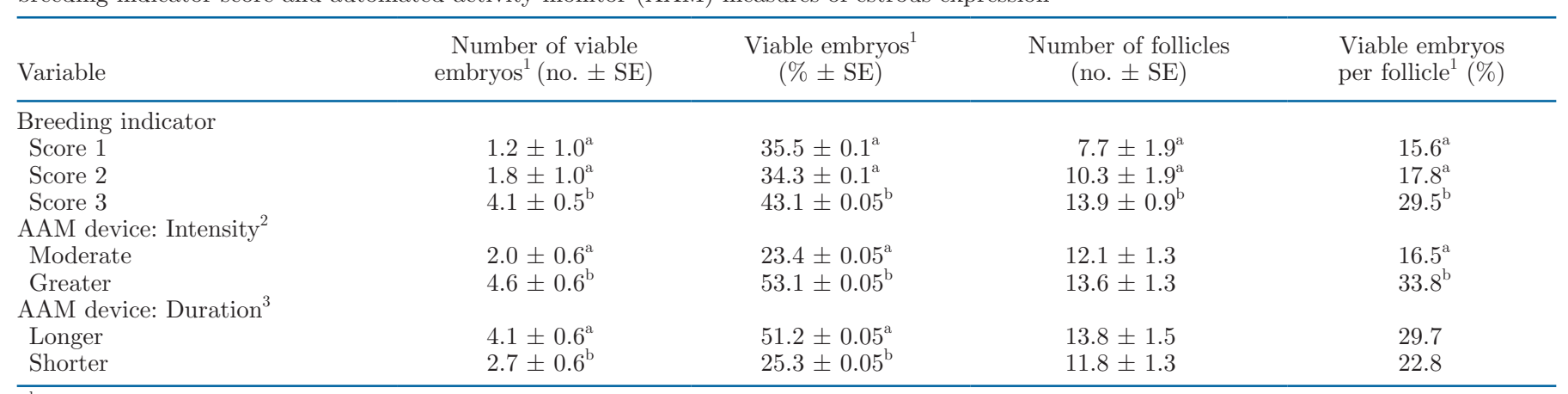

${ }^{\mathrm{a}, \mathrm{b}}$ Different superscript letters indicate differences between variables within columns $(P<0.05)$.

${ }^{1}$ Embryos were considered viable if they were fertilized and of excellent or good quality.

${ }^{2}$ Intensity of estrous expression using the AAM was determined as the maximum activity during an estrus episode and categorized as moderate or greater using the median.

${ }^{3}$ Duration of estrous expression using the AAM was determined as the total time the heifer spent with activity above the threshold and categorized as longer or shorter using the median.

our study, a total of $34.8 \%$ of heifers did not stand to be mounted (score 1 on the breeding indicator) but had an increase in physical activity detected by the AAM. Recent studies have shown that the intensity and duration of the estrus event is associated with ovulation rates and timing, pregnancy per AI, and pregnancy loss (Madureira et al., 2015, 2019; Burnett et al., 2017, 2018; Polsky et al., 2017; Silper et al., 2017), but the physiological mechanisms that explain this link are still unclear.

One possible explanation for decreased estrous behavior close to ovulation may be the abnormal pattern of concentrations of progesterone at and after AI (Madureira et al., 2018). Increased progesterone decreases LH pulses from the anterior pituitary, resulting in lower circulating estradiol concentrations (Stevenson and Pulley, 2016), thus inhibiting or decreasing its effects in the hypothalamic nuclei that trigger modifications in behavior (Lyimo et al., 2000). However, estradiol concentrations do not seem to be highly correlated with increased physical activity as detected by AAM (Lyimo et al., 2000; Madureira et al., 2015). Increased estradiol concentration increases the duration and frequency of standing behavior (Lyimo et al., 2000), but perhaps increased physical activity is dependent on estradiol for its initiation but not for its intensity. Nonetheless, it is remarkable that animals enrolled in and responding to a multiple ovulation protocol do not express estrous behavior, considering the elevated preovulatory plasma concentrations of estradiol (Soumano et al., 1996).

In our study, heifers displaying greater intensity of estrus had a greater proportion of viable embryos. Studies have shown the importance of progesterone in the first week after conception to support embryo and fetal development (Bisinotto et al., 2010; Spencer et al., 2016). In a recent study, Madureira et al. (2019) showed that cows with greater estrous expression had lower concentrations of progesterone at AI and greater concentrations of progesterone $7 \mathrm{~d}$ post-AI. In addition, Pereira et al. (2016) reported that cows that expressed estrus had lower concentrations of progesterone compared with cows that did not express estrus near timed AI. This suggests that the circulating progesterone profile around the time of AI affected both estrous behavior and fertilization and early embryo development.

Total number of follicles was associated with estrous expression as detected by the breeding indicator but not by the AAM. Response to a superovulation protocol is important to optimize the number of fertilized and, ideally, transferable embryos. The total mean $( \pm$ SD) ovulatory response in this study was $67.5 \pm 26.3 \%$. Heifers with a breeding indicator score of 1 had a lower ovulatory response $(42.1 \pm 8.0 \%)$ than did those that had some or all of the indicator rubbed off (i.e., score $3 ; 74.0 \pm 4.9 \% ; P<0.01)$. Variability in superovulation responses have been reported previously (Keller and Teepker, 1990; Kafi and McGowan, 1997; Bó and Mapletoft, 2014), and this variability may be related to the superovulation protocol itself, as well as to other factors related to the animal and environment (Kakar et al., 2005; Chebel et al., 2008).

In conclusion, greater estrous expression was associated with a greater number of embryos collected and a greater percentage of viable embryos. The strength of estrous expression was not associated with the number of follicles present at AI but was associated with a greater ovulatory response. 


\section{ACKNOWLEDGMENTS}

This study was supported by the Dairy Research Cluster Initiative II (Dairy Farmers of Canada, Agriculture and Agri-Food Canada, the Canadian Dairy Network and the Canadian Dairy Commission; Ottawa, Canada). Partial funding was also provided by the Natural Sciences and Engineering Research Council, Discovery Grant (Ottawa, Canada). We thank Fazenda Sekita (Sao Gotardo, Minas Gerais, Brazil) and their farm personnel for contributing to this research project, as well as students, interns and operational support from Conapec Jr., Sao Paulo State University, Botucatu, Brazil. The authors have not stated any conflicts of interest.

\section{REFERENCES}

Bisinotto, R. S., E. S. Ribeiro, L. T. Martins, R. S. Marsola, L. F. Greco, M. G. Favoreto, C. A. Risco, W. W. Thatcher, and J. E. P. Santos. 2010. Effect of interval between induction of ovulation and artificial insemination (AI) and supplemental progesterone for resynchronization on fertility of dairy cows subjected to a 5 -d timed AI program. J. Dairy Sci. 93:5798-5808. https://doi.org/10.3168/ jds.2010-3516.

Bó, G. A., and R. J. Mapletoft. 2014. Historical perspectives and recent research on superovulation in cattle. Theriogenology 81:3848. https://doi.org/10.1016/j.theriogenology.2013.09.020.

Burnett, T. A., A. M. L. Madureira, B. F. Silper, A. C. C. Fernandes, and R. L. A. Cerri. 2017. Integrating an automated activity monitor into an artificial insemination program and the associated risk factors affecting reproductive performance of dairy cows. J. Dairy Sci. 100:5005-5018. https://doi.org/10.3168/jds.2016-12246.

Burnett, T. A., L. Polsky, M. Kaur, and R. L. A. Cerri. 2018. Effect of estrous expression on timing and failure of ovulation of Holstein dairy cows using automated activity monitors. J. Dairy Sci. 101:11310-11320. https://doi.org/10.3168/jds.2018-15151.

Chebel, R. C., D. G. B. Demétrio, and J. Metzger. 2008. Factors affecting success of embryo collection and transfer in large dairy herds. Theriogenology 69:98-106. https://doi.org/10.1016/j .theriogenology.2007.09.008.

Denis-Robichaud, J., S. J. LeBlanc, A. Jones-Bitton, B. F. Silper, and R. L. Aoki Cerri. 2018. Pilot study to evaluate the association between the length of the luteal phase and estrous activity detected by automated activity monitoring in dairy cows. Front. Vet. Sci. 5:210. https://doi.org/10.3389/fvets.2018.00210.

Edmonson, A. J., I. J. Lean, L. D. Weaver, T. Farver, and G. Webster. 1989. A body condition scoring chart for Holstein dairy cows. J. Dairy Sci. 72:68-78. https://doi.org/10.3168/jds.S0022 -0302(89)79081-0.

FASS. 1999. Guide for the Care and Use of Agricultural Animals in Agricultural Research and Teaching. FASS, Champaign, IL.

Hasler, J. F. 2014. Forty years of embryo transfer in cattle: A review focusing on the journal Theriogenology, the growth of the industry in North America, and personal reminisces. Theriogenology 81:152-169. https://doi.org/10.1016/j.theriogenology.2013.09.010.

IETS. 2013. IETS 2012 Statistics and Data Retrieval Committee Report. The year 2011 worldwide statistics of embryo transfer in domestic farm animals. Embryo Transf. Newsletter 2010:14-26.

Jaton, C., A. Koeck, M. Sargolzaei, F. Malchiodi, C. A. Price, F. S. Schenkel, and F. Miglior. 2016. Genetic analysis of superovulatory response of Holstein cows in Canada. J. Dairy Sci. 99:3612-3623. https://doi.org/10.3168/jds.2015-10349.

Jinks, E. M., M. F. Smith, J. A. Atkins, K. G. Pohler, G. A. Perry, M. D. MacNeil, A. J. Roberts, R. C. Waterman, L. J. Alexander, and
T. W. Geary. 2013. Preovulatory estradiol and the establishment and maintenance of pregnancy in suckled beef cows. J. Anim. Sci. 91:1176-1185. https://doi.org/10.2527/jas.2012-5611.

Kafi, M., and M. R. McGowan. 1997. Factors associated with variation in the superovulatory response of cattle. Anim. Reprod. Sci 48:137-157. https://doi.org/10.1016/S0378-4320(97)00033-X.

Kakar, M. A., S. Maddocks, M. F. Lorimer, D. O. Kleemann, S. R. Rudiger, K. M. Hartwich, and S. K. Walker. 2005. The effect of peri-conception nutrition on embryo quality in the superovulated ewe. Theriogenology 64:1090-1103. https://doi.org/10.1016/j .theriogenology.2004.12.017.

Keller, D. S., and G. Teepker. 1990. Effect of variability in response to superovulation on donor cow selection differentials in nucleus breeding schemes. J. Dairy Sci. 73:549-554. https://doi.org/10 .3168/jds.S0022-0302(90)78702-4.

Larimore, E. L., O. L. Amundson, S. L. Bird, B. J. Funnell, S. G. Kruse, G. A. Bridges, and G. A. Perry. 2015. Influence of estrus at fixed-time artificial insemination on early embryonic development in beef cattle. J. Anim. Sci. 93:2806-2812. https://doi.org/ $10.2527 /$ jas.2015-8892.

Lyimo, Z. C., M. Nielen, W. Ouweltjes, T. A. Kruip, and F. J. C. M. van Eerdenburg. 2000. Relationship among estradiol, cortisol and intensity of estrous behavior in dairy cattle. Theriogenology 53:1783-1795. https://doi.org/10.1016/S0093-691X(00)00314-9.

Madureira, A. M. L., T. A. Burnett, J. L. M. Vasconcelos, and R. L. A. Cerri. 2018. Impact of estrous expression on progesterone concentrations and its association with fertility. J. Dairy Sci. 101 (Suppl. 2):83. (Abstr.)

Madureira, A. M. L., L. B. Polsky, T. A. Burnett, B. F. Silper, S. Soriano, A. F. Sica, K. G. Pohler, J. L. M. Vasconcelos, and R. L. A. Cerri. 2019. Intensity of estrus following an estradiol-progesterone-based ovulation synchronization protocol influences fertility outcomes. J. Dairy Sci. 102:3598-3608. https://doi.org/10.3168/ jds.2018-15129.

Madureira, A. M. L., B. F. Silper, T. A. Burnett, L. Polsky, L. H. Cruppe, D. M. Veira, J. L. M. Vasconcelos, and R. L. A. Cerri. 2015. Factors affecting expression of estrus measured by activity monitors and conception risk of lactating dairy cows. J. Dairy Sci 98:7003-7014. https://doi.org/10.3168/jds.2015-9672.

Peippo, J., Z. Machaty, and A. Peter. 2011. Terminologies for the preattachment bovine embryo. Theriogenology 76:1373-1379. https:/ /doi.org/10.1016/j.theriogenology.2011.06.018.

Pereira, M. H. C., M. C. Wiltbank, and J. L. M. Vasconcelos. 2016. Expression of estrus improves fertility and decreases pregnancy losses in lactating dairy cows that receive artificial insemination or embryo transfer. J. Dairy Sci. 99:2237-2247. https://doi.org/10 $.3168 /$ jds.2015-9903.

Polsky, L. B., A. M. L. Madureira, E. L. D. Filho, S. Soriano, A. F. Sica, J. L. M. Vasconcelos, and R. L. A. Cerri. 2017. Association between ambient temperature and humidity, vaginal temperature, and automatic activity monitoring on induced estrus in lactating cows. J. Dairy Sci. 100:8590-8601. https://doi.org/10.3168/ jds.2017-12656.

Rivera, F., C. Narciso, R. Oliveira, R. L. A. Cerri, A. Correa-Calderón, R. C. Chebel, and J. E. P. Santos. 2010. Effect of bovine somatotropin $(500 \mathrm{mg})$ administered at ten-day intervals on ovulatory responses, expression of estrus, and fertility in dairy cows. J. Dairy Sci. 93:1500-1510. https://doi.org/10.3168/jds.2009-2489.

Robertson, I., and R. E. Nelson. 1998. Certification and identification of the embryo. Pages 103-107 in Manual of the International Embryo Transfer Society. 3rd ed. D. A. Stringfellow and S. M. Seidel, ed. Int. Embryo Transfer Soc., Savoy, IL.

Roelofs, J., F. López-Gatius, R. H. F. Hunter, F. J. C. M. van Eerdenburg, and C. Hanzen. 2010. When is a cow in estrus? Clinical and practical aspects. Theriogenology 74:327-344. https://doi.org/10 .1016/j.theriogenology.2010.02.016.

Sartori, R., A. H. Souza, J. N. Guenther, D. Z. Caraviello, L. N. Geiger, J. L. Schenk, and M. C. Wiltbank. 2004. Fertilization rate and embryo quality in superovulated Holstein heifers artificially inseminated with X-sorted or unsorted sperm. Anim. Reprod. 1:86-90. 
Schenk, J. L., T. K. Suh, and G. E. Seidel Jr.. 2006. Embryo production from superovulated cattle following insemination of sexed sperm. Theriogenology 65:299-307. https://doi.org/10.1016/j .theriogenology.2005.04.026.

Silper, B. F., A. M. L. Madureira, L. B. Polsky, S. Soriano, A. F. Sica, J. L. M. Vasconcelos, and R. L. A. Cerri. 2017. Daily lying behavior of lactating Holstein cows during an estrus synchronization protocol and its associations with fertility. J. Dairy Sci. 100:8484-8495. https://doi.org/10.3168/jds.2016-12160.

Soumano, K., D. W. Silversides, F. Doizé, and C. A. Price. 1996. Follicular 33-hydroxysteroid dehydrogenase and cytochromes P450 $17 \alpha$-hydroxylase and aromatase messenger ribonucleic acids in cattle undergoing superovulation. Biol. Reprod. 55:1419-1426. https:/ /doi.org/10.1095/biolreprod55.6.1419.

Spencer, T. E., N. Forde, and P. Lonergan. 2016. The role of progesterone and conceptus-derived factors in uterine biology during early pregnancy in ruminants. J. Dairy Sci. 99:5941-5950. https://doi .org/10.3168/jds.2015-10070.
Stevenson, J. S., and S. L. Pulley. 2016. Feedback effects of estradiol and progesterone on ovulation and fertility of dairy cows after gonadotropin-releasing hormone-induced release of luteinizing hormone. J. Dairy Sci. 99:3003-3015. https://doi.org/10.3168/jds .2015-10091.

Woelders, H., T. van der Lende, A. Kommadath, M.F.W. te Pas, M.A. Smits, and L.M.T.E. Kaal. 2014. Central genomic regulation of the expression of oestrous behaviour in dairy cows: A review. Animal 8:754-764. https://doi.org/10.1017/S1751731114000342.

\section{ORCIDS}

A. M. L. Madureira (ำ https://orcid.org/0000-0001-9696-8478

T. A. Burnett (ㄴ) https://orcid.org/0000-0003-2417-4016

J. L. M. Vasconcelos ๑ https://orcid.org/0000-0001-6324-3614

R. L. A. Cerri ๑ https://orcid.org/0000-0002-8169-8900 